http://jmscr.igmpublication.org/home/ ISSN (e)-2347-176x ISSN (p) 2455-0450 crossref DOI: https://dx.doi.org/10.18535/jmscr/v8i1.52

\title{
Incidental Finding of Jejunal Diverticulum in a Case of Intestinal Obstruction - KMC, Katihar
}

\author{
Authors \\ Dr Khwaja Nasim Ahmad', Dr Md. Abdur Rahman², Dr Yasir Tajdar³, \\ Dr Amjad Zia Mallik ${ }^{4}$, Dr (Major) Md. Mazharul Haque ${ }^{5}$, \\ Dr Ankita Sharma ${ }^{6}$, Dr Shashank Saurav ${ }^{7}$ \\ ${ }^{1}$ Associate Professor, Dept. of Gen. Surgery, KMC, Katihar \\ ${ }^{2}$ Senior Resident, Dept. of Gen. Surgery, KMC, Katihar \\ ${ }^{3}$ Senior Resident, Department of General Surgery, KMC, Katihar \\ ${ }^{4}$ Prof. and H.O.D., Dept. of General Surgery, KMC, Katihar \\ ${ }^{5}$ Associate Prof., Dept. of Gen. Surgery, KMC, Katihar \\ ${ }^{6,7}$ PGT, Department of General Surgery, KMC, Katihar
}

\begin{abstract}
Jejunal diverticulosis is rarely seen in jejunum and clinically present as intestinal obstruction which is very difficult to diagnose pre-operatively and is a per operative finding. We are reporting a case of a 56year old male patient who had clinical symptoms of generalized abdominal pain and vomiting off and on for one and a half year. The patient presented in casualty with signs and symptoms of acute intestinal obstruction with nausea and vomiting. On clinical examination, the abdomen was distended with a central abdominal distension and raised bowel sounds i.e borborygmy. The erect abdominal X-Ray showed multiple air-fluid levels and dilated jejunal loops. Following this, the patient underwent an emergency exploratory laparotomy. Per operatively we found multiple jejunal diverticulae, multiple bands and adhesions involving jejunum and proximal ileum. The adhesions and bands were removed and the part of jejunul diverticulum was resected and anastomosed primarily. Inflamed diverticulae was sent for histopathological examination.

Keywords: jejunal diverticulosis, bowel obstruction, bands, localized cocoon.
\end{abstract}

\section{Introduction}

Jejunal diverticulosis is an uncommon condition seen in $0.5-2.3 \%$ of all small bowel contrast studies, $0.3-4.5 \%$ of autopsies, and $2.3 \%$ of enteroclysis. It is generally a per operative finding which is noticed at the time of exploratory laparotomy. General clinical features of the patients are non-specific symptoms of chronic coliky abdominal pain, diarrhoea, malabsorption, distension, nausea and vomiting. Complications include perforation, diverticulitis, hemorrhage, enterolith formation and intestinal obstruction. Repeated diverticulitis results in formation of bands and adhesions and dynamic intestinal obstruction.

We are reporting a case of multiple jejunal diverticulitis presenting as intestinal obstruction due to formation of multiple bands and adhesions. 


\section{Case Study}

A 56-year old male patient presented in the casualty of K.M.C, Katihar with extensive abdominal pain and multiple episodes of bilious vomiting for last two days. The patient had a history of multiple episodes of pain and occasional fullness in the upper abdomen for the previous two years. On presentation, the patient was found to be hemodynamically stable. Abdominal examination showed distension in the umbilical region and epigastric region with localized tenderness, guarding, rigidity with hyperactive bowel sounds. On per rectal examination we found ballooning with normal anal tone which was not blood stained. Blood examination showed raised TLC, blood urea and serum creatinine. Serum electrolyte was also deranged. Erect and supine abdominal radiographs revealed multiple air fluid levels. A diagnosis of acute intestinal obstruction was made after which an emergency exploratory laparotomy was performed. The largest diverticulum was $4 \times 3 \times 3$ $\mathrm{cm}$ at the mesentric border with multiple adhesions in jejunum and proximal ileum forming a blind loop. A segmental resection of one and half feet of jejunum with end-to-end jejunojejunostomy was performed after removing all the bands and adhesions. The patient's post-operative course was uneventful and the patient was discharged on $13^{\text {th }}$ day after surgery. The histopathological examination of the specimen showed focal mucosal ulceration with submucosal edema and chronic inflammatory infiltrate, whereas multiple sections taken from diverticulae exhibited features of diverticulitis along with an area of stricture.

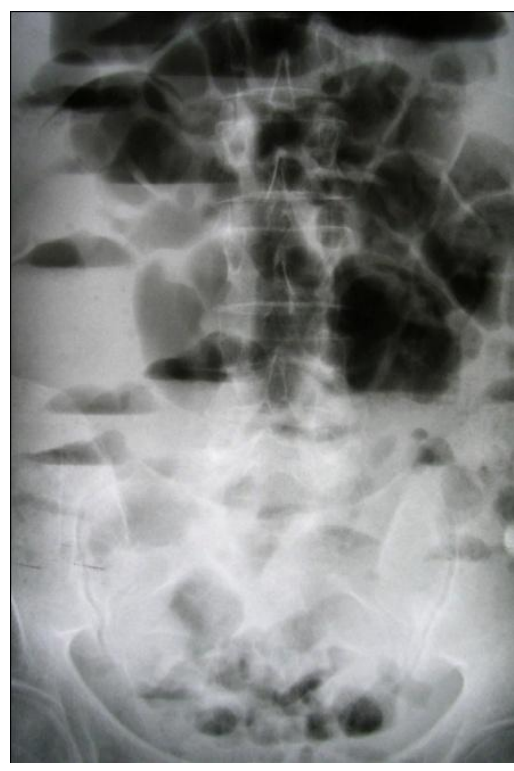

Fig 1

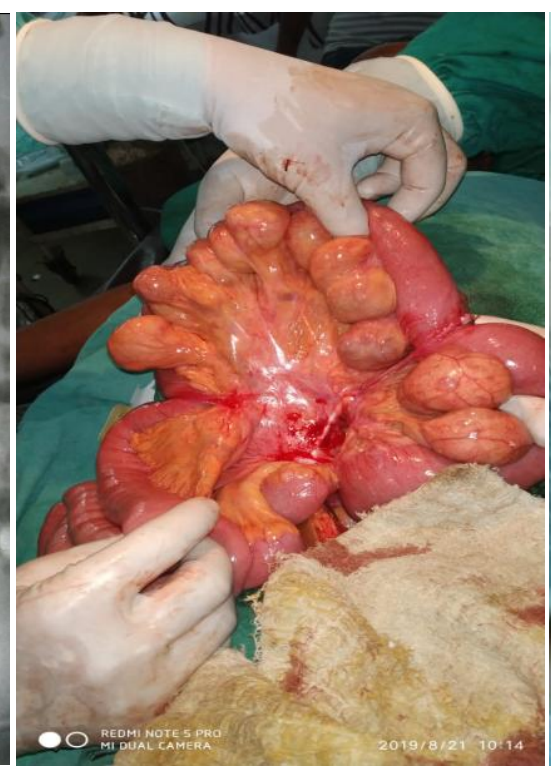

Fig 2

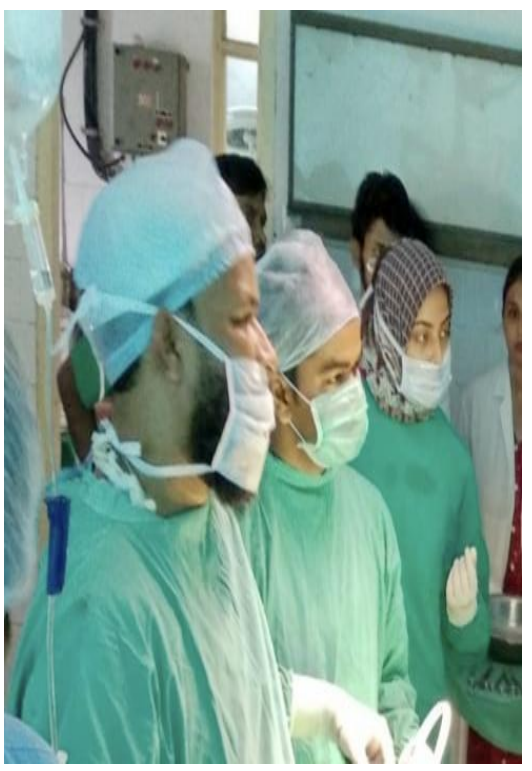

Fig 3

\section{Discussion}

Sommeringsin 1974 described Jejunal diverticulum is an acquired condition first and later by Astley Cooler. It is a false diverticulum characterized by herniation of only mucosa and submucosa in contrast to meckel's diverticulum, and lacks a muscularis propria.The incidence of small bowel diverticulosis is very low compared to colonic diverticulosis that is found in $15-40 \%$ of adults over 40 years of age. Only $0.07-1 \%$ of the population are affected by jejunal and ileal diverticulosis. Jejunal diverticulum is usually asymptomatic until it is associated with complications. The patient presents with chronic abdominal discomfort, bloating, dyspepsia with vague abdominal pain with nausea and vomiting. Complications occur in $15 \%$ of cases and include inflammation, obstruction may be dynamic or adynamic, perforation and hemorrhage. Adynamic intestinal obstruction is most common with jejunal 
diverticulosis occurring as a result of peritonitis, perforations, strangulation or incarceration of enterolith. The recommended treatment for jejunal diverticulum is resection, often performed as emergency surgery, and end-to-end jujuno-jejunal anastomosis to avoid further complications. For symptomatic diverticulum or bleeding diverticulum, the treatment is simple diverticulectomy by wedge resection followed by anastomosis taking precaution that lumen should not get narrowed.

\section{Conclusion}

Jejunal diverticulosis is often clinically asymptomatic with equivocal symptoms in uncomplicated cases resulting in delay in diagnosing the disease. Therefore, its treatment should start with integrated pre-diagnostic approaches like physical abdominal examination, abdominal radiograph and barium meal follow through to assess the patient for possible complications. As soon as the diagnosis is confirmed, a decision to proceed for immediate exploratory laparotomy is made for diverticular resection and surgical removal of bands and adhesions to prevent further complications that could lead to internal hemorrhage, perforation, intestinal obstruction, strangulation, enterolith formation and pus exudation. This relieves the patient and ensures an uneventful post-operative discharge.

\section{Reference}

1. Fischer's Mastery of Surgery $-6^{\text {th }}$ edition.

2. Maingot's Abdominal Operation- $13^{\text {th }}$ edition.

3. BAILEY AND LOVE'S short practice of surgery- $27^{\text {th }}$ edition.

4. Farquharson's text book of operative general surgery- $10^{\text {th }}$ edition.

5. The Washington Manual of surgery- $6^{\text {th }}$ edition.

6. Alam S, Bobby DVM, Balu K. Intestinal Obstruction due to multiple jejunal diverticulae. Indian J Surg 2005; 67:224 [Google Scholar]

7. Lacz NL, Zurlo JV. Small bowel diverticulitis: an often overlooked cause of acute abdomen. Emerg Radiol 2010; 17:497-501 [PubMed] [Google Scholar] 